\title{
Plasmodium spp. membrane glutathione S-transferases: detoxification units and drug targets
}

\author{
Andreas Martin Lisewski* \\ Department of Molecular and Human Genetics, Computational and Integrative Biomedical Research Center, Baylor College of \\ Medicine, Houston, TX 77030, USA. \\ * Corresponding Author: Andreas Martin Lisewski; E-mail: lisewski@bcm.edu
}

\begin{abstract}
Membrane glutathione S-transferases from the class of membrane-associated proteins in eicosanoid and glutathione metabolism (MAPEG) form a superfamily of detoxification enzymes that catalyze the conjugation of reduced glutathione (GSH) to a broad spectrum of xenobiotics and hydrophobic electrophiles. Evolutionarily unrelated to the cytosolic glutathione $S$ transferases, they are found across bacterial and eukaryotic domains, for example in mammals, plants, fungi and bacteria in which significant levels of glutathione are maintained. Species of genus Plasmodium, the unicellular protozoa that are commonly known as malaria parasites, do actively support glutathione homeostasis and maintain its metabolism throughout their complex parasitic life cycle. In humans and in other mammals, the asexual intraerythrocytic stage of malaria, when the parasite feeds on hemoglobin, grows and eventually asexually replicates inside infected red blood cells (RBCs), is directly associated with host disease symptoms and during this critical stage GSH protects the host RBC and the parasite against oxidative stress from parasite-induced hemoglobin catabolism. In line with these observations, several GSH-dependent Plasmodium enzymes have been characterized including glutathione reductases, thioredoxins, glyoxalases, glutaredoxins and glutathione Stransferases (GSTs); furthermore, GSH itself have been found to associate spontaneously and to degrade free heme and its hydroxide, hematin, which are the main cytotoxic byproducts of hemoglobin catabolism. However, despite the apparent importance of glutathione metabolism for the parasite, no membrane associated glutathione S-transferases of genus Plasmodium have
\end{abstract}

been previously described. We recently reported the first examples of MAPEG members among Plasmodium spp.

One reason why Plasmodium MAPEG enzymes might have been missed in the past is the fact that there is no significant sequence homology between known MAPEGs and Plasmodium protein sequences. This is not unexpected given that the genome sequence of the human malaria parasite Plasmodium falciparum, the most studied Plasmodium species and the causative agent of the most severe form of malaria in humans, still lacks functional annotations for most of its genes. To address this problem, we developed a network-based functional prediction method with increased sensitivity over traditional sequence homology based methods (termed graph-based information diffusion on compressed supergenomic networks) that integrates evolutionary and interaction-specific links over hundreds of genomes. Among many produced predictions, the method pointed to the small parasitophorous vacuolar membrane antigen PfEXP1 (Plasmodium falciparum exported protein 1) as having GST activity. This possibility warranted further investigation because Pfexp 1 was already known as one of the most highly transcribed genes during ring and trophozoite stages of falciparum malaria, and because it occupies a locus which has resisted gene disruption attempts and thus indicated its essentiality.

We confirmed the computational prediction experimentally: after heterologous expression in E. coli GST activity of purified PfEXP1 toward the standard substrate 1chloro-2,4-dinitrobenzene (CDNB) was measured. The link to malaria parasite biology was further strengthened by our next observation that PfEXP1 as well as its close ortholog Plasmodium yoelii PyHEP17 (17 kDa hepatocyte

MICROREVIEW on: Lisewski AM, Quiros JP, Ng CL, Adikesavan AK, Miura K, Putluri N, Eastman RT, Scanfeld D, Regenbogen SJ, Altenhofen L, Llinás M, Sreekumar A, Long C, Fidock DA and Lichtarge $O$ (2014). Supergenomic network compression and the discovery of EXP1 as a glutathione transferase Inhibited by artesunate. Cell 158(4):916-28. doi: 10.1016/j.cell.2014.07.011 
protein) both use hematin as a substrate, i.e. they conjugate reduced glutathione onto hematin to form hematinGSH adducts. Thus far, these enzymes appear to be the only known MAPEG GSTs with hematin substrate specificity (Figure 1) and may therefore enable the parasite to buffer oxidative stress produced through excess hematin. This protective activity may be important during the ring stages of the parasite, when PfEXP1 levels are high, and when hemoglobin catabolism has already started but when significant hematin biocrystals (hemozoin) buildup and the formation of a main food vacuole have yet to begin. Interestingly, these ring stages have been also described as highly sensitive to artemisinins, the family of sesquiterpene lactones that contain a peroxide bridge, and which today form the frontline treatment against malaria. Because of the large body of evidence that artemisinins require a hemoglobin degradation product, possibly heme or hematin, as the molecular activator of their peroxide bridge and thus of their full antimalarial action, we hypothesized that artemisinins might inhibit the membrane GST activity of PfEXP1 in a hematin dependent manner. In vitro inhibition studies confirmed that in the presence of free hematin artesunate (a water soluble artemisinin derivative) is a potent and competitive inhibitor with a half maximal inhibitory concentration near $1 \mathrm{nM}$. Without hematin, artesunate inhibition of PfEXP1 GST activity toward CDNB was 100 -fold less potent and uncompetitive. This strong hematin dependence may explain why PfEXP1, which is also expressed during host liver stages when parasite in- duced hemoglobin degradation does not yet take place, would be targeted by artemisinins specifically after RBC invasion.

Competitive inhibition of PfEXP1 through artesunate opened the possibility that PfEXP1 might play a role in the metabolic degradation of artesunate, and we observed that PfEXP1 indeed facilitates the conjugation of GSH with artesunate (Figure 1) both in vitro and in parasites cultured under selective pressure through dihydroartemisinin. These selected parasites, which recrudesce earlier and with a larger population after exposure to high levels of artemisinins than their drug-sensitive parental strains, show differential expression of Pfexp 1 at mRNA and at protein levels. In contrast to the membrane bound PfEXP1, the cytosolic PfGST lacks this catalytic ability, is not differentially expressed in these more resistant strains, and thus is probably incapable to modulate parasite susceptibility to artemisinins. Interestingly, other common antimalarial drugs such as chloroquine and atovaquone are not catalytically conjugated onto GSH through PfEXP1 (our unpublished data.) Thus PfEXP1 appears to be involved in a metabolic degradation pathway specific to artemisinins which can be upregulated in drug pressured parasites that are less sensitive to artemisinin. These results suggest that membrane associated GSTs in Plasmodium spp. are relevant to the recently observed loss of artemisinin efficacy in field and clinical studies. This loss has been primarily evidenced through longer times it takes to clear parasites from patient blood, and Plasmodium membrane GSTs may consti-

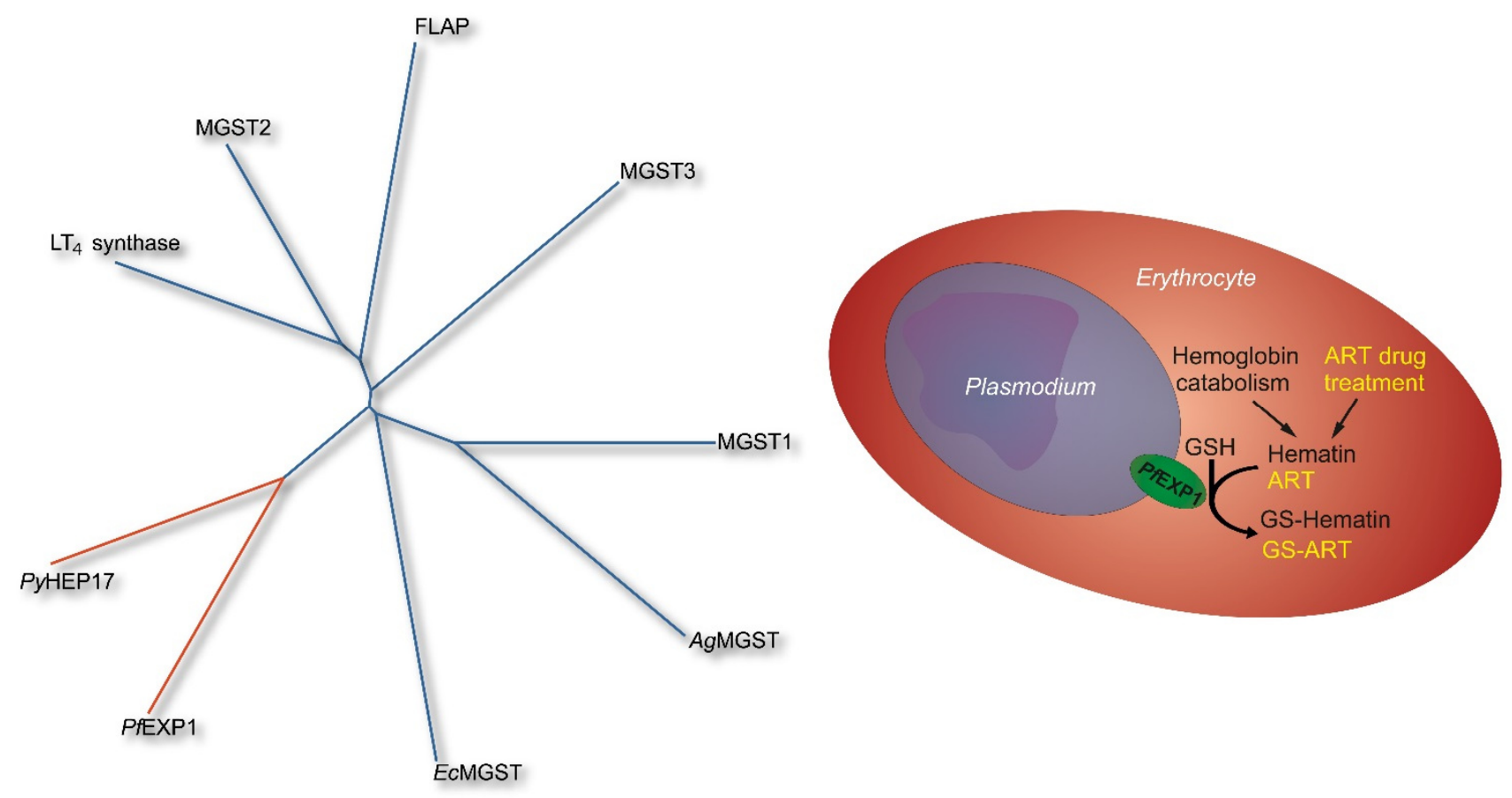

FIGURE 1: (Left) Representative dendrogram of the MAPEG superfamily including the malaria parasite GSTs Plasmodium yoelii HEP17 (PyHEP17) and Plasmodium falciparum EXP1 (PfEXP1), Escherichia coli MGST (EcMGST), Anopheles gambiae MGST (AgMGST), rat MGST1, and human MGST3, 5-lipoxygenase activating protein (FLAP), MGST2, and leukotriene C4 synthase. (Right) Proposed model of PfEXP1 in Plasmodium falciparum infected erythrocytes. The membrane protein PfEXP1 catalyzes the conjugation of reduced glutathione (GSH) onto hematin and onto the drug artemisinin (ART) and thus contributes to their detoxification. 
tute a significant part of a parasite defense system to withstand exposure to artemisinins.

\section{ACKNOWLEDGMENTS}

Financial support was provided through the National Science Foundation grants DBI-1356569 and CCF-0905536.

\section{CONFLICT OF INTEREST}

The author declares no conflicts of interest.

\section{COPYRIGHT}

(C) 2014 Lisewski. This is an open-access article released under the terms of the Creative Commons Attribution (CC BY) license, which allows the unrestricted use, distribution, and reproduction in any medium, provided the original author and source are acknowledged.

Please cite this article as: Andreas Martin Lisewski (2014). Plasmodium spp. membrane glutathione S-transferases: detoxification units and drug targets. Microbial Cell 1(11): 387389. doi: 10.15698/mic2014.11.177 